\title{
Herpes Simplex Virus-2 (HSV-2) Type-specific Antibody Correlates of Protection in Infants Exposed to HSV-2 at Birth
}

\author{
Rhoda L. Ashley, * Julie Dalessio, * Sandra Burchett, ${ }^{\star}$ Zane Brown, ${ }^{*}$ Sylvia Berry, Kathey Mohan, * and Lawrence Corey *\| \\ Departments of ${ }^{*}$ Laboratory Medicine, ${ }^{\ddagger}$ Pediatrics, ${ }^{\S}$ Obstetrics and Gynecology, and "Medicine, \\ University of Washington, Seattle, Washington 98105
}

\begin{abstract}
Western blot analysis was used to compare the herpes simplex virus (HSV)-2 antibody profiles of 40 infants $<2$ wk of age who had been exposed to maternal genital HSV-2 at birth. 4 mothers were HSV seronegative at delivery and seroconverted to HSV-2 ("primary infection"), 9 had HSV-1 antibodies and seroconverted to HSV-2 ("nonprimary first episode infection"), and 27 were HSV-2 seropositive ("recurrent infection"). Neonatal herpes infections developed in 1 of 4 infants of women with primary infection, in 3 of 9 infants of women with nonprimary first episode infection, and in none of the 27 infants of women with recurrent HSV-2. Antibodies to HSV-2 proteins gG-2, VP5, and ICP35 were detected in 83,89 , and $72 \%$ of the 36 uninfected infants, respectively. None of the four infected infants had detectable antibodies to gG-2 and only one (25\%) had antibodies to VP5 or ICP35. The more limited profiles of the 13 infants born to mothers with first episodes of HSV-2 were then analyzed separately; these profiles were similar among infected and uninfected infants except for gG-2, which elicits antibodies that are type specific for HSV-2. None of the infected infants versus seven of nine $(\mathbf{7 8 \%})$ uninfected infants were gG-2 seropositive. These comparisons suggest that maternal type-specific antibodies may play a role in preventing neonatal infection after exposure to HSV-2. (J. Clin. Invest. 1992. 90:511-514.) Key words: neonatal • herpes hominis • serology • transplacental immunity
\end{abstract}

\section{Introduction}

Previous studies have shown an inverse correlation between titers of passively acquired maternal neutralizing antibody and acquisition of infection in the neonate (1-3). High levels of acquired antibody have been associated with milder outcomes after neonatal infection in some cohorts $(1,4,5)$ but not all (6). In neonatal mice the presence of antibodies that mediate antibody-dependent cell cytotoxicity (ADCC) ${ }^{1}$ and neutraliza-

A portion of the data in this manuscript was presented at the XVI International Herpesvirus Workshop, 7-12 July 1991, Asilomar, CA, and appeared in abstract form (Abstract 7B-02).

Address correspondence to Rhoda Ashley, Ph. D., University of Washington, Virology Division, c/o Children's Hospital and Medical Center, D-536, 4800 Sand Point Way NE, Seattle, WA 98105.

Received for publication 19 September 1991 and in revised form 10 January 1992.

1. Abbreviations used in this paper: ADCC, antibody-dependent cell cytotoxicity; HSV, herpes simplex virus.

J. Clin. Invest.

(C) The American Society for Clinical Investigation, Inc.

$0021-9738 / 92 / 08 / 0511 / 04 \$ 2.00$

Volume 90, August 1992, 511-514 tion are associated with protection against viral challenge (7, 8 ). Neutralizing antibody activity and ADCC activity are primarily directed against herpes simplex virus (HSV) glycoproteins (9). The viral glycoprotein targets of these protective antibody activities are now being elucidated at the epitope level in the mouse model (10).

In a recent prospective study, we showed that infants born to mothers with first episodes of genital HSV-2 had a markedly higher incidence of infection than those born to mothers with recurrent HSV-2 infections, suggesting that type-specific antibodies are particularly important in protecting the neonate after exposure (11). Women who acquire HSV-2 close to term and who are seropositive for HSV-1 have an equal risk of transmitting to their neonates as do seronegative mothers with primary HSV-2 infections at term (11). This finding suggests that passively transferred maternal HSV-1 antibodies confer little protection against neonatal HSV-2 infection. Sullender and his colleagues (12) have reported a higher prevalence of antibody to one HSV-2 glycoprotein, gG-2, in healthy infants exposed to HSV-2 at birth than in infants infected at birth. The possible protective effect of passively transferred antibodies to other HSV-2 proteins remains unclear. Therefore, we used a sensitive Western blot method to describe the passively transferred antibody profiles of 40 infants exposed to HSV-2 at birth. Nine major HSV-2 protein targets were identified in these profiles and the prevalence of antibodies to these proteins was compared between infected and uninfected infants as well as between infants whose mothers had first episode HSV-2 infections and those whose mothers had recurrent HSV-2 episodes at term.

\section{Methods}

Study population. Infants exposed to HSV-2 at delivery were identified as part of a prospective study of all parturient women without clinical evidence of genital herpes who entered the University of Washington Medical Center or a private community hospital between 1982 and 1989 (11). Cervical and vulvar specimens were obtained for viral culture. Of 15,923 mothers with viral cultures who delivered live born infants within $48 \mathrm{~h}$ of culture, $56(0.35 \%)$ had HSV isolated from their genital tracts at entry into the delivery suite. 51 of the 56 had HSV-2 and 5 had HSV-1 by monoclonal antibody subtyping (13). All infants exposed to HSV were immediately recalled for viral cultures and serum collection. Of the 51 infants exposed to HSV-2, 40 had sera available for testing. Maternal serum was also drawn at $6 \mathrm{wk}$ after delivery. Sera were coded and frozen at $-20^{\circ} \mathrm{C}$.

Neonatal HSV infection was defined as the isolation of HSV from the neonate after $24 \mathrm{~h}$ of life. All such infants were immediately started on systemic antiviral therapy according to the guidelines of the National Institute of Allergy and Infectious Diseases Neonatal HSV Study Group (14). Of the $\mathbf{4 0}$ infants on this study, 4 developed HSV-2 infections and 36 remained uninfected.

Western blot analysis. HSV-2-infected cell proteins were electrophoretically separated and transferred as described previously $(15,16)$. 
Briefly, detergent lysate of infected cells was denatured and electrophoresed for $1.5 \mathrm{~h}$ at $25 \mathrm{~mA}$ per gel in a minigel apparatus (Hoefer Scientific Instruments, San Francisco, CA ) then transferred to nitrocellulose (Schleïcher and Schuell, Keene, NH) in Towbin buffer for $2 \mathrm{~h}$ at $200 \mathrm{~mA}$ in a Transphor apparatus (Hoefer Scientific Instruments). Nitrocellulose was cut into strips ("blots") for the solid-phase immunoassay.

A modification of the immunostaining protocol was used to increase sensitivity and decrease the volume of sera needed for the test. Briefly, sera were diluted 1:200 in $1 \mathrm{ml}$ of Blotto (1\% nonfat milk in PBS) and incubated at room temperature overnight with individual HSV-2 blots. Strips were then washed with PBS containing $0.5 \%$ Tween 20, rinsed with PBS, and incubated $1.5 \mathrm{~h}$ with horseradish peroxidase-conjugated goat anti-human IgG (diluted 1:10,000 in PBS containing 4\% goat serum; Boehringer Mannheim Biochemicals, Indianapolis, IN). After washing and rinsing as above, TMB Membrane Peroxidase Substrate (3', 3, 5', 5-tetramethylbenzidine; Kirkegaard Perry Laboratories, Gaithersburg, MD) was mixed according to the manufacturer's instructions and added to the blots for $5 \mathrm{~min}$. The reaction was stopped by rinsing with distilled water.

All sera were tested on the same day using the same lot of HSV-2 Western blots. Additional blots were stained using mouse monoclonal antibodies to gG, VP16, and gD. Reactive bands were detected with horseradish peroxidase-conjugated goat anti-mouse IgG (1:1,000 in PBS; Boehringer Mannheim Biochemicals) to confirm the identity of immunoreactive bands (17). Glycoprotein gI was identified by the relative migration characteristics of a glycosylated protein (18); the identity of this band is, therefore, considered preliminary. Nucleocapsid proteins VP5 and ICP35 were identified by their lack of glycosylation and by their migration characteristics in bis-acrylamide crosslinked gels (19). Sera were coded so that bands were scored without knowledge of either infant outcome or maternal serostatus.

Statistical methods. Fisher's exact analysis was used to analyze differences in prevalence of antibodies between infants who developed infections and those who did not. Chi square with Yates' correction was used to analyze antibody prevalence data between infants of mothers with recurrent HSV-2 infection and those whose mothers had first episode HSV-2 infection at delivery.

\section{Results}

Classification of maternal HSV-2 infections. All 40 mothers had HSV-2 isolated from genital secretions sampled in the 48 hours before delivery. Comparison of the HSV serologic profiles of the mother at the time of delivery and postpartum revealed seroconversion to HSV-2 in 13 and persistently stable HSV-2 antibodies in 27.4 of the 13 women who seroconverted postpartum had either no HSV antibodies or evidence of early HSV-2 seroconversion ("primary infection"). Nine women had a full complement of HSV-1 antibodies in their entry sera and seroconverted to HSV-2 ("nonprimary first episode HSV2 "). One of the four infants born to a mother with primary HSV-2 subsequently developed neonatal herpes as did three of the nine infants born to mothers with nonprimary first episode HSV-2 infections. The remaining 27 mothers had HSV-2 antibodies on entry into the delivery room, shed HSV-2 at delivery, and had unchanged Western blot profiles at postnatal followup indicating they had reactivation of latent genital HSV-2 infections at term. None of the infants of these 27 mothers with recurrent HSV-2 episodes developed neonatal herpes.

Prevalence of $H S V-2$ protein-specific antibodies in infants. To evaluate the association between HSV-2 antibodies and disease acquisition, we analyzed the Western blot profiles from the first available infant sera. The time from delivery to collection of sera was $1-7 \mathrm{~d}$ in 28 infants, $8-14 \mathrm{~d}$ in 10 , and $15 \mathrm{~d}$ in 2 infants (median $5.5 \mathrm{~d}$ ). Onset of infection occurred at 3, 5, and $11 \mathrm{~d}$ of age in three neonates. A fourth was culture positive one day after delivery but did not develop symptoms before being lost to follow-up on day 4. Among the three symptomatic infected infants, sera were tested at 3,7 , and $14 \mathrm{~d}$ of life, respectively. The fourth, asymptomatic infant had serum collected at day 3 of life before his release to care in another city.

When the 13 infants born to mothers with first episode HSV-2 infections were analyzed, the incidence of antibodies to $\mathrm{VP5}, \mathrm{gB}, \mathrm{gC}, \mathrm{gE}, \mathrm{gI}, \mathrm{VP} 16, \mathrm{gD}$, and ICP35 did not differ signifcantly between infected and uninfected infants $(P \geq 0.05)$ ( Table I). However, infected infants had a significantly lower incidence of antibodies to gG-2 ( 0 of 4 versus 7 of $9 ; P<0.05$ ). As expected, the 27 infants born to mothers with recurrent infection had a significantly higher prevalence of antibodies to VP5 $(P<0.0001)$, gG $(P<0.001), \mathrm{gC}(P<0.0005), \mathrm{gD}(P$ $=0.05)$, and ICP35 $(P<0.0001)$ than did the 13 born to mothers who had first episode infection at term. Of interest, the incidence of antibodies to HSV-2 gB, gE, gI, and VP16 were not significantly different between infants born to mothers with recurrent or first episode HSV-2 infections $(P>0.05)$.

When the prevalence values of the 4 infected and 36 uninfected infants from the entire study population were compared, the most dramatic differences were seen in the prevalence of antibodies to gG-2, VP5, and ICP35. None of the infected infants had detectable antibodies to gG-2 while 34 of $36(94 \%)$ uninfected infants had gG-2 antibodies. 1 of $4(25 \%)$ infected infants had antibodies to VP5 or ICP35 versus 29 of $36(81 \%)$ uninfected infants.

Antibody profiles in infected versus uninfected infants. Passively transferred antibody profiles were very limited in infants born to mothers with primary first episode HSV-2 infections (Fig. $1 A$ ). The infected infant from this group had detectable antibodies against $\mathrm{gB}, \mathrm{gE}$, and $\mathrm{gD}$ (Fig. $1 A$, lane 1 ). Two other infants born to mothers with primary infections had antibodies

Table I. Antibody Profiles from Infants with Maternal History of First Episode or Recurrent HSV-2 Genital Infections

\begin{tabular}{|c|c|c|c|}
\hline \multirow{4}{*}{$\begin{array}{l}\text { HSV-2 } \\
\text { protein }\end{array}$} & \multicolumn{3}{|c|}{ Number of infants with antibody to HSV-2 protein (\%) } \\
\hline & Maternal first episode & Maternal first episode & Maternal recurrent episode \\
\hline & Infant infected & Infant not infected & Infant not infected \\
\hline & $n=4$ & $n=9$ & $n=27$ \\
\hline VP5 & $1(25)$ & $3(33)$ & $27(100)^{*}$ \\
\hline gB & $4(100)$ & $6(67)$ & $21(78)$ \\
\hline gG-2 & $0(0)$ & $7(78)$ & $27(100)^{*}$ \\
\hline $\mathrm{gC}$ & $2(50)$ & $4(44)^{\ddagger}$ & $27(100)^{*}$ \\
\hline gE & $2(50)$ & $4(44)$ & $22(81)$ \\
\hline gI & $1(25)$ & $2(22)$ & $9(33)$ \\
\hline VP16 & $2(50)$ & $7(78)$ & $19(70)$ \\
\hline $\mathrm{gD}$ & $3(75)$ & $7(78)$ & $27(100)^{5}$ \\
\hline ICP35 & $1(25)$ & $3(33)$ & $26(96)^{*}$ \\
\hline
\end{tabular}

* $P<0.001$ by Chi square with Yates' correction comparing antibody prevalences of infants born to mothers with recurrent episodes ( $n$ $=27$ ) and antibody prevalence of infants whose mothers had first episode HSV-2 infection $(n=13)$. ${ }^{\ddagger} P<0.05$ by Fisher's exact test comparing antibody prevalence in infected $(n=4)$ versus uninfected $(n=9)$ infants born to women with first episode HSV-2 infections. ${ }^{\S} P=0.05$ by Chi square with Yates' correction between infants of recurrent mothers and infants of mothers with first episodes. 

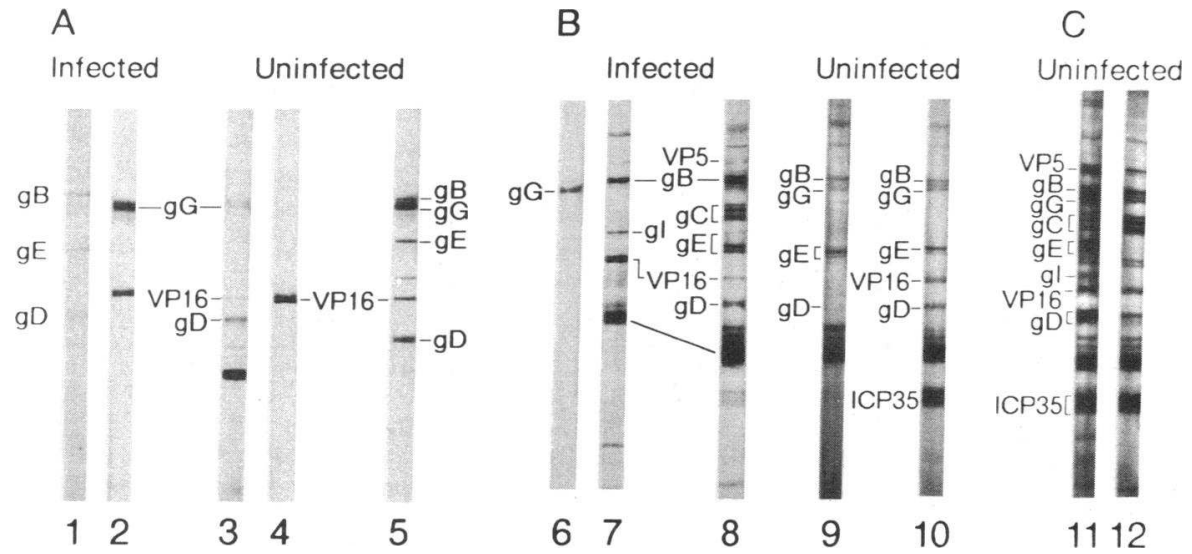

Figure 1. Antibody profiles from infected and uninfected infants. Antibody profiles from infected infants (lanes 1,7 , and 8 ) were compared with those of uninfected infants (lanes 3, 5, and 9-12). Infants from mothers with primary HSV-2 infections $(A)$, nonprimary first episode HSV-2 infections $(B)$, and recurrent HSV-2 infections $(C)$, reflect increasing levels of reactive antibodies. Multiple gels were required for this study; blots from each gel were reacted with monoclonal antibodies to gG2 (lanes 2 and 6 ) and VP16 (lane 4) to localize proteins on profles from the respective gels.

to gG-2, VP16, and gD (Fig. $1 A$, lanes 3 and 5). The fourth infant had no detectable antibodies against HSV-2 proteins and remained uninfected.

The HSV-2 antibody profiles of infants born to women with nonprimary first episodes had more bands than were seen with sera from infants born to mothers who had primary infections (Fig. $1 \mathrm{~B}$ ). Profiles from two of the three infected infants born to women with nonprimary first episodes of HSV-2 include antibodies to gB, VP16, (lanes 7 and 8 ) and, in one case, several other viral proteins (lane 8 ). Neither had detectable antibody to gG-2. Profiles from two uninfected babies revealed antibodies to gB, gG-2, gE, and gD (lanes 9 and 10). Antibodies to other proteins such as VP5, VP16, and ICP35 were present in some, but not all, sera (Fig. $1 B$, lane 10 , and Table I).

Antibody profiles in infants born to women with recurrent genital HSV-2 infections reflected the passive transfer of abundant antibodies to a large number of viral protein targets (Fig. 1 $C$, lanes 11 and 12). No differences in number of HSV-2 protein targets were observed in antibody profiles of infants with both HSV-1 and HSV-2 passive antibodies and infants with only HSV-2 antibodies (data not shown).

\section{Discussion}

Two aspects of this trial are unique: the ability to identify and test infants exposed to HSV-2 at birth and the ability to identify passive antibody to nine major HSV-2 immunogens in infants who did or did not develop HSV-2 infections after exposure. We used Western blot techniques to determine whether passive antibody profiles differed in the 4 infants who did and the 36 infants who did not develop herpes infections after exposure to HSV-2 at birth. We found that the prevalence of detectable antibody to most HSV-2 proteins was similar between infected and uninfected babies. However, antibodies to gG-2 were less likely to be detected in samples from the infected infants as compared with uninfected infants. Our previous studies of antibody responses in adults have shown that antibodies to $\mathrm{gG}-2$, as detected by Western blot, do not arise as early during seroconversion as do antibodies to $\mathrm{gB}$ or $\mathrm{gD}(20)$. Since all of the infected infants were born to mothers who were in the process of seroconverting to HSV-2, these infants would be less likely to have these "late" antibodies to gG-2. However, when infected $(n=4)$ versus uninfected $(n=9)$ infants of first episode, seroconverting mothers were compared, the marked difference in antibody prevalence to $\mathrm{gG}-2$ remained ( $0 \%$ versus $78 \%$ for infected versus uninfected, respectively).

Detectable antibody to gG-2 was highly correlated with protection from developing infection after HSV-2 exposure. Of 13 infants whose mothers were seroconverting to HSV-2, seven had antibodies to gG-2; none became infected. Of the six infants who were seronegative for $\mathrm{gG}-2$, four $(67 \%)$ became infected while two (33\%) remained uninfected. While it is possible that the six gG-2 seronegative infants may have had antibody levels too low for detection by our Western blot assay, our data indicate a definite quantitative difference in the antibodies passively transferred to infected versus uninfected infants. Because IgG class antibody has a half-life of $\sim 21 \mathrm{~d}$, it is unlikely that the apparent differences in $\mathrm{gG}-2$ antibody prevalence were due to decay of maternal antibody. Our findings extend those of Sullender et al. using an enzyme immunoassay for gG-2 in which 30 of $34(88 \%)$ infants exposed to HSV-2 at birth had gG-2 antibody and remained well while one infant who developed neonatal herpes lacked antibody to gG-2 (12).

Protective factors other than detectable levels of passively transferred antibody to gG-2 were clearly operative for the two infants in our study and the four infants in Sullender's study who were gG-2 seronegative and uninfected. Human to mouse adoptive transfer studies have shown that protective immunity against HSV infection is a multifactorial system involving macrophages, $\mathrm{CD}_{-4}$ lymphocytes, lymphokines including IFNgamma and IL-2, and antibody (21). The role of antibody in this model may be via an antibody-dependent cell cytotoxicity function (8). High titers of maternally derived ADCC antibodies have been associated with protection in infants exposed to $\mathrm{HSV}$ at birth and to local versus disseminated infections in those infants who developed disease (22). Antibodies to gG-2 have not been shown to have either neutralizing or ADCC activity in vitro.

It is possible that $\mathrm{gG}-2$ antibodies have served in our Western blot system as markers of more subtle type-specific antibodies with neutralizing, ADCC, or other biologic functions directly involved in preventing infection. Both $\mathrm{gB}$ and $\mathrm{gD}$, for example, have neutralizing and ADCC functions. Antibodies elicited by HSV-1 infections but directed against type-common epitopes make it difficult to detect type-specific seroconversion to HSV-2 gB and gD by Western blot (23). In this study, for example, of nine infants born to HSV-1 seropositive mothers who were seroconverting to HSV-2, six (67\%) had detectable antibody that reacted with HSV-2 gB and seven (78\%) had 
antibody reactive with $\mathrm{HSV}-2 \mathrm{gD}$. These antibodies could be to either type-common epitopes or to type-specific epitopes; Western blot cannot discern antibodies to type-specific epitopes, nor can it detect antibodies to conformational epitopes that may have biologic significance. Clearly, the relative roles of antibodies to type-specific or type-common epitopes of $\mathrm{gG}-2, \mathrm{gB}, \mathrm{gD}$, or other HSV-2 proteins will be determined only with assays based on type-specific epitopes from individual proteins.

While evidence from animal models confirms the protective effect of passively transferred antibodies to individual proteins (10), the protective effect depends upon the dose of challenge virus and the relative competence of other arms of the immune system. The absence of maternally derived antibody to gG-2 may define a group of infants at higher risk of infection after HSV-2 exposure. Yet there is no evidence that antibodies to gG-2 alone prevent infection. Maternal virus titer, length of time of virus exposure, and the presence of genital mucosal antibodies to HSV (24), are other factors which may affect transmission. The role of serum antibody in preventing infection in the human neonate remains an important question because antiviral therapy has not been completely effective in preventing morbidity and mortality of symptomatic herpes infections in the neonate. Immunoprophylaxis regimens are an appealing potential option for exposed neonates. Our work suggests that antibodies to type-specific epitopes (which predominate on gG-2) may be of particular value in immunoprophylaxis. Identification of other protein- and epitope-specific antibodies that block transmission is necessary to combine optimal antibody components for protective passive immunization.

\section{Acknowledgments}

This work was supported by Herpes Program Project grant AI 30731 to L. Corey and by a March of Dimes Birth Defects Foundation grant to Z. Brown.

\section{References}

1. Yeager, A. S., A. M. Arvin, L. J. Urbani, and J. A. Kemp. 1980. Relationship of antibody to outcome in neonatal herpes simplex virus infections. Infect. Immun. 29:532-538.

2. Yeager, A. S., and A. M. Arvin. 1984. Reasons for absence of a history of recurrent genital infections in mothers of neonates infected with herpes simplex virus. Pediatrics. 73:188-193.

3. Prober, C. G., W. M. Sullender, L. L. Yasukawa, D. S. Au, A. S. Yeager, and A. M. Arvin. 1987. Low risk of herpes simplex virus infection in neonates exposed to the virus at the time of vaginal delivery to mothers with recurrent genital herpes simplex virus infection. N. Engl. J. Med. 316:240-244.

4. Sullender, W. M., J. L. Miller, L. L. Yasukawa, J. S. Bradley, S. B. Black, A. S. Yeager, and A. M. Arvin. 1987. Humoral and cell-mediated immunity in neonates with herpes simplex virus infection. J. Infect. Dis. 155:28-37.

5. Kohl, S., M. S. West, C. G. Prober, L. S. Loo, W. Sullender, and A. Arvin. 1989. Neonatal antibody-dependent cellular cytotoxicity antibody levels are asso- ciated with the clinical presentation of neonatal herpes simplex virus infection. $J$. Infect. Dis. 160:770-776.

6. Whitley, R. J., A. J. Nahmias, A. M. Visintine, C. L. Fleming, and C. A. Alford. 1980. The natural history of herpes simplex virus infection of mother and newborn. Pediatrics. 66:489-494.

7. Baron, S., M. G. Worthington, J. Williams, and J. W. Gaines. 1976. Post-exposure serum prophylaxis of neonatal Herpes Simplex infection of mice. Nature (Lond.). 261:505-506.

8. Kohl, S., and L. S. Loo. 1982. Protection of neonatal mice against herpes simplex virus infection: probable in vivo antibody-dependent cellular-cytotoxicity. J. Immunol. 129:370-376.

9. Norrild, B., S. Shore, and A. Nahmias. 1979. Herpes simplex virus glycoproteins: participation of individual herpes simplex virus type 1 glycoprotein antigens in immunocytolysis and their correlation with previously identified glycoprotein. J. Virol. 32:741-748.

10. Kohl, S., N. Strynadka, R. Hodges, and L. Pereira. 1990. Analysis of the role of antibody-dependent cellular cytotoxic antibody activity in murine neonatal herpes simplex virus infection with antibodies to synthetic peptides of glycoprotein D and monoclonal antibodies to glycoprotein B. J. Clin. Invest. 86:273278.

11. Brown, Z. A., J. Benedetti, R. L. Ashley, S. Burchett, S. Selke, S. Berry, L. A. Vontver, and L. Corey. 1991. Neonatal herpes simplex virus infection in relation to asymptomatic maternal infection at the time of labor. $N$. Engl. J. Med. 324:1247-1252.

12. Sullender, W. M., L. L. Yasukawa, M. Schwartz, L. Pereira, P. A. Hensleigh, C. G. Prober, and A. M. Arvin. 1988. Type-specific antibodies to herpes simplex virus type 2 (HSV-2) glycoprotein $G$ in pregnant women, infants exposed to maternal HSV-2 infection at delivery, and infants with neonatal herpes. J. Infect. Dis. 157:164-171.

13. Petersen, E. P., O. W. Schmidt, L. C. Goldstein, R. C. Nowinski, and L. Corey. 1983. Typing of clinical HSV isolates using mouse monoclonal antibodies to HSV-1 and HSV-2: comparison with type-specific rabbit antisera and restriction endonuclease analysis of viral DNA. J. Clin. Microbiol. 17:92-96.

14. Whitley, R., A. Arvin, C. Prober, S. Burchett, L. Corey, D. Powell, S Plotkin, S. Starr, C. Alford, J. Connor, et al. 1991. A controlled trial comparing vidarabine with acyclovir in neonatal herpes simplex virus infection. $N$. Engl. J. Med. 324:444-449.

15. Ashley, R. L., J. Militoni, F. Lee, A. Nahmias, and L. Corey. 1988. Comparison of Western blot (immunoblot) and glycoprotein G-specific immunodot enzyme assay for detecting antibodies to herpes simplex virus types 1 \& 2 in human sera. J. Clin. Microbiol. 26:662-667.

16. Ashley, R. L. 1989. Genital Herpes Infections. In Clinics in Laboratory Medicine: Sexually Transmitted Diseases. Vol. 9. F. N. Judson, editor. W. B. Saunders Co., Philadelphia, PA. 405-420.

17. Ashley, R., K. Mack, M. Shurtleff, C. Critchlow, and L. Corey. 1988 Differential effect of systemic acyclovir treatment of genital HSV-2 infections on antibody responses to individual HSV-2 proteins. J. Med. Virol. 4908:703-708.

18. Longnecker, R., S. Chatterjee, R. J. Whitley, and B. Roizman. 1987. Identification of a herpes simplex virus 1 glycoprotein gene within a gene cluster dispensable for growth in cell culture. Proc. Natl. Acad. Sci. USA. 84:4303-4307.

19. Braun, D. K, B. Roizman, and L. Pereira. 1984. Characterization of post-translational products of herpes simplex virus gene 35 proteins binding to the surfaces of full capsids but not empty capsids. J. Virol. 49:142-153.

20. Lopez, C., A. Arvin, and R. L. Ashley. 1992. Immunity to herpesvirus infections in man. In The Human Herpesviruses: Biology, Pathogenesis, and Treatment. B. Roizman and C. Lopez, editors. Raven Press, New York.

21. Kohl, S., L. S. Loo, and S. B. Greenberg. 1982. Protection of newborn mice from a lethal herpes simplex virus infection by human interferon, antibody, and leukocytes. J. Immunol. 128:1107-1111.

22. Kohl, S. 1989. The neonatal human's immune response to herpes simplex virus infection: a critical review. Pediatr. Infect. Dis. J. 8:67-74.

23. Ashley, R., and J. Militoni. 1988. Use of densitometric analysis for interpreting HSV serologies based on Western blot. J. Infect. Dis. 157:319-326.

24. Ashley, R. L., J. Militoni, P. Wilson, M. Remington, A. Dunn, and L. Corey. 1990. Protein targets of the humoral and mucosal IgA responses to herpes simplex virus 2 (HSV-2). Program Abstr. 30th Interscience Conference on Antimicrobial Agents and Chemotherapy, 21-24 Oct., 1990. 198:119 A. (Abstr.) 